\title{
Gerhard Stickel
}

\section{Zur deutschen Sprache: die Innensicht der Außensicht}

\begin{abstract}
Germanisten aus dem Sprachinland sind auch an der Außenansicht ihres sprachlichen Gegenstands interessiert. Um einen Eindruck von Ansichten der deutschen Sprache aus Perspektiven des Sprachauslands zu gewinnen, wurden Mitglieder des Internationalen Wissenschaftlichen Rats des IDS gebeten, Einstellungen und (stereotype) Meinungen mitzuteilen, die in ihren Ländern zur deutschen Sprache und zu den Deutschen verbreitet sind. Die Ergebnisse dieser kleinen Umfrage werden zusammenfassend vorgestellt und kommentiert. Insgesamt ist das Bild, das sich dabei vom Deutschen und seinen Sprechern ergibt, wenig erfreulich. Auch einige alte Vorurteile erweisen sich als beständig. Aber es gibt auch ermutigende Relativierungen einiger negativer Bewertungen und einige bemerkenswert positive Ausnahmen.
\end{abstract}

\section{Persönlicher Einstieg}

Ich beginne mit eigenen Erfahrungen. Noch als Student sah ich mich gern als Weltbürger, der mit humanistischen Ideen ausgestattet überall in der Welt zu Hause sein könnte. Die ersten Jahre nach der Nazizeit und dem Kriegsende waren ja für einen jungen Deutschen nicht gerade dazu angetan, gerne deutsch sein zu wollen. Kürzere Reisen ins europäische Ausland änderten daran nur wenig. Erst drei Semester an einer amerikanischen Universität machten mir nach und nach deutlich, wie deutsch ich trotz aller kosmopolitischen Neigungen war, und das nicht nur in meinen Ansichten, meinem Verhalten, sondern auch in meiner Sprache, die nun keine alltägliche Selbstverständlichkeit mehr war. Im anderssprachigen alltäglichen Lebenskontext war die eigene Sprache zum bemerkenswert Besonderen geworden.

Noch stärker wurde ich auf die deutsche Sprache aufmerksam bei einem zweieinhalbjährigen Aufenthalt an einer japanischen Universität. Auch dort machte mir die sprachliche Normalität des japanischen Alltags die eigene Sprache zwar nicht fremd, ließ mich aber ihre relativen Besonderheiten deutlicher erfahren. Verstärkt wurde dies durch die Aufgabe, Deutsch an junge Japaner zu vermitteln. Dabei habe auch ich mit den Studenten viel Deutsch gelernt: nicht das aktuale Sprechen und Schreiben - das konnte ich ja schon ganz gut - sondern als bewusste Wahrnehmung von formalen Regeln, semantischen Differenzen und Gebrauchskonventionen. So ist mir zum Beispiel die kuriose Semantik der Verben kommen und gehen klar geworden, die 
es uns erlaubt, zu einem Adressaten nicht nur zu sagen Komm bitte zu mir!, sondern auch Ich komme gleich zu dir, dass also kommen die Bewegung zum Sprecher bezeichnen kann, aber auch die auf den Adressaten oder dritte Ziele hin. Gelernt habe ich dies aus fehlerhaften deutschen Äußerungen der Studenten $(*$ Ich gehe morgen zu Ihnen) und nachdem ich einige Male die japanischen Verben kuru und iku analog zu kommen und gehen gebraucht und damit Unverständnis erzeugt hatte; denn im Japanischen kann man zu einem Adressaten nur, gehen', nicht aber ,kommen'. Der Blick von außen, über den Vergleich mit einer anderen Sprache, hat hierbei einen kleinen Bereich der eigenen Sprache deutlicher erkennen lassen.

\section{Motive für das Tagungsthema „Deutsch von außen“}

Es lohnt sich also für Linguisten, die sich mit ihrer eigenen Sprache als Forschungsgegenstand befassen, sich auch um die Außenansicht dieser Sprache zu kümmern, weil dies die Beschränkung auf die muttersprachliche Binnenperspektive überwinden hilft, weil es durch den Vergleich die methodisch nützliche Distanz zum Gegenstand schafft. Die Distanz zur eigenen Sprache ist bekanntlich gerade dann schwer zu gewinnen, wenn sie nicht nur Objektsprache, sondern auch Beschreibungssprache ist. Dieses methodische Problem haben zumindest die deutschsprachigen Germanisten, die im wesentlichen monolingual aufgewachsen sind. Und das sind derzeit wohl noch die meisten.

Kurzum, die Erweiterung und Schärfung der Innensicht durch die Außensicht war das Hauptmotiv des Instituts für Deutsche Sprache zur Wahl des Tagungsthemas „Deutsch von außen“. Es war auch schon einer der Gründe für eine Reihe von kontrastiv-linguistischen Projekten zu verschiedenen Sprachenpaaren, die über die Jahre hin am Institut oder mit Unterstützung des IDS durchgeführt worden sind. ' Auch für die hier dokumentierte Tagung erhofften wir uns unter anderem von Germanisten aus dem nichtdeutschsprachigen Ausland Beobachtungen und Feststellungen zu dem gemeinsamen Gegenstand aus der Außenperspektive. Eine vergleichende Betrachtung ist der sogenannten Auslandsgermanistik geradezu natürlich, weil Aneignung und Vermittlung von Deutsch als Fremdsprache und der in ihr verfassten Literatur ausgesprochene oder implizite Vergleiche mit der jeweils eige-

1 Zemb, Jean-Marie: Vergleichende Grammatik Französisch-Deutsch, 2 Bde., Mannheim/Wien/Zürich 1978, 1984. Engel, Ulrich/Mrazović, Pavica (Hrsg.): Kontrastive Grammatik deutsch-serbokroatisch. 2 Bde., München/Novi Sad 1986. Kaneko, Tohru/ Stickel, Gerhard (Hrsg.): Deutsch und Japanisch im Kontrast, Bde. 1, 2 u. 4, Heidelberg $1983 \mathrm{ff}$. Cartagena, Nelson/Gauger, Hans Martin: Vergleichende Grammatik Spanisch-Deutsch, 2 Bde., Mannheim/Wien/Zürich 1989. Engel, Ulrich/Isbasescu, Mihai/ Stanescu, Speranta/Nicolae, Octavian: Kontrastive Grammatik deutsch-rumänisch, 2 Bde., Heidelberg 1993. Engel, Ulrich et al.: Deutsch-polnische kontrastive Grammatik, 2 Bde., Heidelberg 1999. 
nen Sprache und Literatur besonders nahelegen. Unser eigenes Motiv für das Thema „Deutsch von außen“ traf sich mit dem wiederholt geäußerten Wunsch von ausländischen Mitgliedern unseres Internationalen Wissenschaftlichen Rats, ihre Sicht auf die deutsche Sprache darstellen und diskutieren zu können, einschließlich der Rolle, die das Deutsche auch als Unterrichtsgegenstand in ihren jeweiligen Heimatländern hat.

\section{Außenperspektiven}

Den anderen Vorträgen, die während der Tagung gehalten und diskutiert wurden und die in diesem Band wiedergegeben werden, soll nun ein kleines Präludium vorausgeschickt werden, und zwar mit einer kommentierten $\mathrm{Zu}$ sammenstellung mehr oder weniger fester Meinungen zur deutschen Sprache, über die in den Wochen vor der Tagung Kolleginnen und Kollegen aus verschiedenen Ländern berichtetet haben.

Von einigen Meinungen und Vorurteilen über das Deutsche hat man auch als Inlandsgermanist schon gehört. Die alte Behauptung Mark Twains etwa, Englisch könne man in 30 Stunden, Französisch in 30 Tagen und Deutsch erst in $30 \mathrm{Jahren}^{\mathrm{lernen}}{ }^{2}$, haben manche von uns bis zum Überdruss oft zitiert gehört, wobei der Überdruss weniger der negativen Einschätzung der Lernbarkeit des Deutschen als der Zitathäufigkeit gilt.

Um einen ersten Überblick über die Außensichten auf das Deutsche zu gewinnen, habe ich mich an eine Reihe von präsumptiven Tagungsteilnehmern gewandt, die nicht schon als Referenten gewonnen waren, und sie um Antworten zu vier Fragen gebeten:

1. Welche pauschalen Meinungen gibt es in Ihrem Land zur deutschen Sprache? (z. B., dass sie schwierig, nützlich, schön, hässlich usw. ist)

2. Welche Eigenschaften werden der deutschen Sprache zugeschrieben? (z. B. ,gutturale' Aussprache, komplizierte Wortstellung, lange Wörter)

3. Gibt es (auch erfundene) Kennwörter oder Merkwörter, die als typisch deutsch gelten?

4. Gibt es stereotype Vorstellungen davon, wie sich ein Deutscher sprachlich verhält?

Experten in empirischer Sozialforschung hätten sicherlich Einiges an diesen Fragen auszusetzen (etwa, dass die ersten beiden Fragen in ihren Zielen nicht deutlich zu trennen sind). Und eine naheliegende Kritik von Linguisten könnte sein, dass ich auch nach Verbotenem gefragt habe. Werturteile, dass eine Sprache nützlich oder gar, dass sie schön oder hässlich sei, sind ja linguistisch verboten, wie auch die Bewertung von Sprachen nach der Schwierigkeit, sie zu lernen, von Sprachdidaktikern nicht gern gesehen wird. Tat-

${ }^{2}$ Mark Twain: A Tramp Abroad, Appendix D. The awful German language. New York 1880. 
sache ist aber, dass es Meinungen und Wertungen dieser Art gibt und dass sie u. a. Einfluss darauf haben, ob Deutsch als Fremdsprache in der Schule oder Germanistik als Studienfach an der Universität gewählt wird. Mir kam es deshalb bei der kleinen Umfrage nicht auf Expertenurteile an, sondern auf die Ermittlung verbreiteter Laienmeinungen. Den ausländischen Adressaten hatte ich darum auch geschrieben, dass es mir in erster Linie um Meinungen und Stereotype von Studierenden und Laien gehe, die möglicherweise vom Deutschen nur wenig oder gar nichts wüssten.

Antworten habe ich von 44 Kolleginnen und Kollegen aus 15 europäischen und 2 außereuropäischen Ländern erhalten. Dafür danke ich auch hier noch einmal ganz herzlich. Und ich bitte um Nachsicht dafür, dass die Antworten im Folgenden nur in Ausschnitten und beschränkt auf das sog. Sprachausland referiert werden. Ein in quantitativer Hinsicht repräsentatives Bild kann auf diese Weise nicht gewonnen werden. Belanglos sind die Antworten dennoch nicht; denn sie kommen von professionellen Germanisten, d. h. Fachleuten für die deutsche Sprache, welche die in ihren Ländern verbreiteten Meinungen über ihren Lehr- und Forschungsgegenstand besonders aufmerksam und sensibel registrieren. Einige von ihnen habe ihre Eindrücke sogar durch gezielte Befragung ihrer Studenten oder Kollegen überprüft. Der polnische Kollege Rafal Szubert hat sich zu einer breit angelegten Fragebogenaktion an Schulen und Hochschulen anregen lassen, die gesondert ausgewertet werden muss, weil bis zum Tagungsbeginn noch nicht alle Ergebnisse vorlagen. Ich bin auch auf eingehende empirische Studien hingewiesen worden wie die gerade erschienene Untersuchung von Marion Perrefort (Besançon) ${ }^{3}$, die ich in diesem Zusammenhang leider nicht berücksichtigen kann. Hier sei nur knapp zusammengefasst, was meine kleine Umfrage an Bemerkenswertem ergeben hat.

\section{1 $\mathrm{Zu}$ pauschalen Bewertungen der deutschen Sprache}

Deutsch gilt generell als „schwierige“, als „schwere“ Sprache. Das wird in fast allen Antworten unabhängig von deren Herkunft bestätigt. Typisch ist die Antwort des französischen Kollegen Réne Metrich (Nancy): „Dass [Deutsch] schwer sei, meinen, soweit ich sehe, ALLE Franzosen - mit Ausnahme der Deutschlehrer, die aus ,strategischen Gründen' ihre Schwierigkeit herunterzuspielen suchen ...“. Deutsch gilt als schwierig auch im Vergleich zum Englischen. Jarmo Korhonen (Helsinki) berichtet, für viele Finnen sei Englisch ,nicht nur eine leichtere, sondern auch ein schönere Sprache als Deutsch“. Und litauische Studenten erklären: „Die deutsche Sprache ist sowohl schwierig als auch nützlich. Viele Leute in Litauen meinen, sie ist schwieriger als das Englische oder Französische." (laut Ernesta Raciene, Vilnius). Das Heterostereotyp von der schweren deutschen Sprache

${ }^{3}$ Perrefort, Marion: J'aimerais aimer parler allemand. Paris 2001. 
ist ja längst zum Autostereotyp der Deutschen geworden. Es gibt wohl auch objektivierbare Eigenschaften, die besonders den ersten Zugang zur deutschen Sprache schwer machen, ganz gleich von welcher Erstsprache aus. Hierzu ist dem Beitrag von Werner Abraham (in diesem Band) einiges zu entnehmen.

Unterschiedlich sind die Bewertungen des Deutschen nach seinem Nutzen. „Schwierig, aber nützlich“ ist eine Formel, die mehrere Kollegen gebrauchen, so etwa Henning Bergenholtz (Arhus) in Dänemark. Dass Deutsch in Finnland für nützlich erachtet wird, vermelden mehrere Kollegen von dort. Sein Nutzen wird besonders für den Bereich des innereuropäischen Handels gesehen. Sein literarischer Wert ist anscheinend weniger gefragt. Wie Ahti Jäntti (Jyväskylä) schreibt: „Deutsch als Sprache der Belletristik ist nicht besonders präsent." Auch in Spanien gelten Deutschkenntnisse als nützlich für Handel und Industrie, aber auch für Philosophen, wenn auch als völlig ungeeignet für moderne Musik (Luisa Schilling, Madrid). In Polen wird der Wert des Deutschen vor allem in seinem Nutzen für den Handel gesehen (Joana Golonka/Rzeszów, Tomasz Czarnecki/Danzig, Alfred Tarantowicz, Lodz). Auch aus Litauen heißt es: „Deutsch ist nützlich, weil man jetzt viele wirtschaftliche Beziehungen zu deutschen Firmen hat." (Ernesta Raciene) In Indien gilt Deutsch als nützlich für die Naturwissenschaften und den Tourismus (Pawan Surana, Jaipur). Japaner bewerten Deutsch als ,gut für naturwissenschaftliche, medizinische und philosophische Schriften" (so Günter Zobel, Tokio). Haruo Nitta (Tokio) schränkt aber ein, die Meinung, Deutsch sei die „Sprache der Wissenschaft und der Philosophen“, werde „heute nicht mehr so viel vertreten wie früher". Heute gelte Deutsch eher ,als schwierig zu lernen“ und als ,nicht mehr so international“.

Als gering wird der praktische Nutzen von Deutsch unter anderem in Frankreich erachtet. Durchweg wird Deutsch für weniger nützlich gehalten als Englisch, zumal in manchen Ländern die Meinung verbreitet ist, man komme in Deutschland auch mit Englisch zurecht. Wie Gottfried R. Marschall (Paris) schreibt, werde in Frankreich von Studenten und Geschäftsleuten mit Deutschlanderfahrung der Eindruck vermittelt, „dass man sich in Deutschland mit Englisch sehr gut verständigen kann, zum einen, weil sowieso ,alle' Deutschen Englisch können, und zweitens weil die deutsche Sprache inzwischen sehr viele englische Wörter aufgenommen hat und die Deutschen einen ,unverständlichen Mischmasch aus Deutsch und Englisch reden“. Aus der Türkei referiert Vural Ülkü (Mersin) die Ansicht: „Deutsch ist sehr nützlich für bestimmte Fachgebiete ... und für Türken, die Verbindung zu Deutschland haben - aber es ist nützlicher, Englisch zu lernen“. Die generelle Bewertung des Deutschen scheint vor allem unter der ältereren Generation in der Türkei positiv zu sein, auch wenn uns die positiv gesehenen Attribute vielleicht nicht behagen. Wie Herr Ülkü schreibt, meinen ältere Türken: „Deutsch ist schneidig, forsch und zackig; Französisch ist für Damen und Künstler, Englisch für Geschäftsleute, Deutsch für echte Männer! 
..., „,militärische Befehle haben in keiner Sprache einen besseren Klang.“ Auch in Bosnien meint die ältere Generation, Deutsch eigne sich besonders für militärische Kommandos und andere Befehle. Ansonsten gelte es als Sprache von Handel, Handwerk und Philosophie (so Miloje Dordevic, Sarajevo). Der militärische Nutzen des Deutschen wird freilich in vielen Ländern weniger geschätzt.

Mit der Schönheit des Deutschen ist es auch nicht weit her. In mehreren Ländern ist offensichtlich die Ansicht verbreitet, Deutsch sei vielleicht nützlich, aber nicht schön, wobei der Schönheitsmangel oder gar die Hässlichkeit vor allem mit phonischen Eigenschaften begründet wird, die deutschen Äußerungen nachgesagt werden. In der Wahrnehmung der meisten Franzosen sei Deutsch ,eine Sprache, die ,gespuckt', bzw. ,gebellt' wird (,une langue qui se crache, qui s'aboie')" (R. Metrich). Dies ginge auf „Reminiszenzen aus dem Krieg“ und auf Kriegsfilme zurück. Als ,unschön, hart, unmelodisch“" gilt Deutsch vielen Italienern (so Marina Foschi und Marianne Hepp, Pisa), und auch die spanischen Studierenden von Marisa Siguan (Barcelona) meinen, Deutsch klinge ,hässlich, trocken, kalt“, aber nur, so schränken einige ein, ,wenn man es nicht kennt“. Die meisten der finnischen Studenten, die Henrik Nikula (Turku) befragt hat, ,finden die Sprache eher hässlich bzw. unschön ...". Deutsch werde für ,,schwierig, hässlich, hart, aggressiv“ gehalten, meint auch Kari Keinästö (Turku). Jozef Wiktorowicz (Warschau) nimmt an, dass es ,unter polnischen Lernern keine ästhetischen Prädikate in Bezug auf die deutsche Sprache“ gebe, Joanna Golonka (Rzeszów) glaubt dagegen, die deutsche Sprache werde in Polen von den meisten als sehr hart, herrisch, laut, ja ,kläffend' empfunden. Diejenigen, die „,sie schön finden, gehören eher zu einer kleinen Minderheit. In Deutsch zu singen finden viele geradezu unästhetisch." Auf ähnliche Meinungen ist Ewa Drewnowska-Vargáné (Veszprem) bei ihren ungarischen Studenten gestoßen: „Deutsch klingt hart. Man sagt, Lieder klingen in deutscher Sprache nicht schön, merkwürdig." Günter Zobel fasst die Meinung mancher Japaner zusammen: Deutsch wirke „militärisch, stabil, eckig, langweilig, liedhaft“. Zur musikalischen Eignung des Deutschen sind offensichtlich die Meinungen geteilt. Auf den Zusammenhang zwischen mangelnder Schönheit der deutschen Sprache und dem Sprachverhalten von Deutschen gehe ich gleich noch ein.

Pauschale Bewertungen des Deutschen werden in einzelnen Zuschriften auch in Verbindung mit der mancherorts noch vorherrschenden Art der Vermittlung gesehen. Deutsch gelte in Italien auch als schwierig, weil es meist noch „traditionell“ unterrichtet werde „mit Aufmerksamkeit auf sämtliche Regeln und Ausnahmen" (M. Foschi/M. Hepp). In Schweden werde der Deutschunterricht als „langweilig“ empfunden, ,weil viele Texte nur von Krieg und Elend handeln" (Christine Palm Meister, Uppsala). Dies erinnert an den Vorwurf, der zeitweilig auch dem sog. ,kritischen' Deutschunterricht der Goethe-Institute in manchen Ländern gemacht worden ist. 
3.2 Zu den formalen Eigenschaften des Deutschen aus der Sicht anderer Sprachen

Solche Eigenschaften sind durchweg im Zusammenhang mit der Pauschalbewertung von Deutsch als schwerer Sprache zu sehen. Ich kann aus den besonders reichhaltigen Antworten hierzu nur eine kleine Auswahl bieten. Die Schwierigkeiten, die das Deutsche anderssprachigen Lernern bereitet, werden je nach Ausgangssprache etwas unterschiedlich begründet, meist aber an grammatischen Spezifika festgemacht. Als Lern- und Verstehenshindernisse werden in fast allen Antworten genannt: Wortstellung und lange Wörter'. Bei der Satztopologie ist es vor allem die Stellung der verbalen Konstituenten (Verbalklammer mit infiniten Verbformen bzw. Finitum am Satzende) und in Verbindung damit auch die Vorfeldbesetzung: „Da steht immer etwas an erster Stelle des Satzes und man weiß nie warum" (R. Metrich). Mit den langen Wörter sind komplexe Komposita gemeint, die Lernern ganz unterschiedlicher Ausgangssprachen Probleme bereiten, aber auch als „kompakte Ausdrucksmöglichkeiten“ (M. Siguan) geschätzt werden.

Von slawischen Sprachen aus, vom Litauischen, Hindi und dem ebenfalls artikellosen Türkischen wird die Erschwernis durch die Artikel genannt. Die deutsche Flexionsmorphologie wird von verschiedenen Sprachen aus als sehr schwierig betrachtet (Ungarisch, Japanisch, Italienisch, Finnisch, Hindi). Aus französischer Sicht wird dann noch hingewiesen auf ,diese verflixten Abtönungspartikeln, ohne die kein Satz richtig deutsch ist." (G. R. Marschall) Vural Ülkü fasst mehrere grammatische Schwierigkeiten zusammen: „Die komplizierte Wortstellung, die unregelmäßigen Verben, die Schachtelsätze und die trennbaren Verben sind eine richtige Folter für die Deutsch lernenden Türken." Laut Alfred Tarantowicz gelten polnischen Deutschlernern „,drei Vergangenheitstempora als überflüssig und unverständlich“. Von Kollegen aus verschiedenen Ländern wird auch auf die schwierige Orthographie des Deutschen ${ }^{4}$ und auf Ausspracheprobleme hingewiesen, auf den ,Konsonantenreichtum, die vielen s-Laute, die Achs und Ichs“ (Ahti Jäntti). Ähnliches wird aus der Sicht des Spanischen (Luisa Schilling), des Japanischen (Haruo Nitta) und des nicht gerade konsonantenarmen Serbokroatischen (Miloje Dordevic) bemerkt. Aus polnischer Sicht weist Jan Czochralski (Warschau) noch auf die Diphthonge und den schwierigen Wortakzent hin. Das Adjektiv guttural hatte ich schon mit meiner Frage vorgegeben. Es wird von mehreren meiner Korrespondenten für das Deutsche übernommen und oft noch durch hart ergänzt. Wie spanische Kollegen berichten, wird der phonetische Eindruck deutscher Wörter karrikiert mit einem fiktiven deutschen Wort für ,Straßenbahn“ oder ,U-Bahn'. Es lautet subanestrujenbajen. Das

${ }^{4}$ „Diskrepanz zwischen dem Schriftbild und der Aussprache, mehrere Zeichen für einen Laut ... Die ansonsten ordnungsliebenden Deutschen haben hier versagt ...". (Miloje Dordevic) 
geht offensichtlich sowohl gegen die langen deutschen Wörter wie auch gegen den Ach-Laut.

\subsection{Zu den Kenn- und Merkwörtern}

Damit sind wir schon bei der 3. Frage, nämlich nach Kenn- und Merkwörtern, also Ausdrücken, die als typisch deutsch gelten. Die Antworten hierzu machen eine Zusammenfassung schwer, was sicherlich an meiner ungenauen Frage lag, die auch auf das weite Feld der Germanismen in den einzelnen Sprachen bezogen werden konnte. ${ }^{5}$ Dennoch erscheint mir einiges als mitteilenswert.

Typisch deutsche Wörter sind aus französischer Sicht u. a. (streng) verboten und kolossal (R. Metrich), in Italien: raus, jawohl, Auf Wiedersehn, Kartoffel, Wurst, Kraut und arbeiten (M. Foschi/M. Hepp). Hinzu kommen auch aus anderen Ländern erwähnte Wörter wie Weltanschauung, Leitmotiv, Kindergarten, Hinterland, Realpolitik, Panzer und Blitz (Dorothee Heller, Bergamo), die es offensichtlich zu Internationalismen gebracht haben. Finnische Studenten nennen u. a. Sauerkraut, Lederhosen, Bier, Achtung, Scheiße, jawohl, kaputt, wunderbar, außerdem noch Besserwisser, Nazi, Führer und SS und Markennamen deutscher Autor (A. Jäntti, J. Korhonen, H. Nikula, Kari Keinästö). In Litauen kennt man u. a. Bier, Scheiße, kaputt, Butterbrot, prima und Schnaps. Beispiele für typisch deutsche Wörter aus spanischer Sicht sind (wen wundert's): Kartoffel, Bratwurst, Sauerkraut, Bier, Oktoberfest und Achtung! Alarm! Halt!, die letzteren wohl aus Comics und Kriegsfilmen (so L. Schilling/M. Siguan). Kommandoworte werden auch in Polen als typisch deutsch angesehen: Halt! Raus! Los! Schnell! außerdem: Ordnung muss sein. (J. Golonka). Hinzu kommen u. a. Klappe zu und Hände hoch! (A. Tarantowicz). Aber auch für das heutige Polen wird die Kenntnis solcher Ausdrücke von den dortigen Kollegen eher auf Kriegsfilme, denn auf eigene Kriegserfahrungen zurückgeführt. Ordnung muss sein führen auch Odleif Leirbukt (Bergen) und Peter Schröder (Halden) als Beispiel aus Norwegen an. Aus Bosnien werden Bitte und Danke genannt (Miloje Dordevic). Als typisch deutsch aus japanischer Sicht nennt Günter Zobel einige in Japan gebrauchsübliche Germanismen wie energishu (,energisch'), arubaito (,Arbeit ${ }^{\text {' }}=$ Nebentätigkeit) und karute $\left(, K^{2}\right.$ arte $=$ Krankenblatt). H. Nitta erwähnt noch Geist und Romantik. Solche eher bildungsprachliche Wörter werden auch aus anderen Ländern genannt. ${ }^{6}$ In der Türkei (V. Ülkü) gelten als typisch deutsche Ausdrücke: ja-nein,

${ }^{5}$ Siehe hierzu etwa die detaillierte Darstellung von Erwin Koller (Braga): Zu den Germanismen im Portugiesischen. In: Ästhetik der Texte - Varietät von Sprache. Festschrift für Jürgen-Schmidt-Radefeldt zum 60. Geburtstag. Tübingen 2000. S. 185-210. Ewa Drewsnowska-Vargáné übermittelte brieflich eine Liste mit Germanismen im Ungarischen wie firhang (,Vorhang'), sámli (,Schemel'), slágvort (,Schlagwort') und andere.

${ }^{6}$ aus Finnland: Angst, Weltanschauung, Weltschmerz, Zeitgeist, Lied, Stillleben, Edelweiß, Finnlandisierung, ins Blaue hinein, anno dazumal, leben und leben lassen (Jarmo Korhonen); aus der Türkei: Mutter, Wolke, danke, bitte, schön, Geist, Gemütlich- 
Ordnung, Disziplin, streng(stens) verboten, untersagt, Formular ausfüllen sowie Wörter mit anlautendem ,Z“ (Zug, Zwanzig, zwei, ...) und anlautendem „sch“ (schreiben, Schlag, Stein, Straße, Spiel ...).

Interessant sind - neben diesen meist mit kulturellen oder historischen Klischeevorstellungen verbundenen realen deutschen Wörtern - erfundene Ausdrücke, die deutsch wirken sollen. Erwähnt habe ich schon das spanisch-deutsche Subanestrujenbajen. In italienischen Comics sprechen deutsche SS-Leute italienische Verben und Substantive mit dem als deutsch empfundenen Suffix -en, also mangiaren (statt manjare, ,essen') oder victorien (statt victoria, ,Sieg'), so der Hinweis der Kolleginnen Foschi und Hepp aus Pisa. ${ }^{7}$ Türkische Kabarettisten, die einen komischen oder bösen Deutschen verkörpern, schreien ein fiktives Deutsch aus ,Wörtern' mit -ingen oder -lingen wie z. B.: „Komm, du Türklingen nix arbeitingen, faulingen, krankingen, urlaubingen, du!!! “ (so V. Ülkü). Dahinter steht offensichtlich nicht nur eine bestimmte Wahrnehmung der deutschen Morphologie, sondern auch der deutschen Arbeitswelt, wie sie sich türkischen Migranten darstellt.

\section{4 $\mathrm{Zu}$ den stereotypen Meinungen vom Sprachverhalten eines Deutschen}

Schon bei den generellen Eigenschaften, die dem Deutschen zugeschrieben werden, ist nicht immer zu unterscheiden, ob sie der Sprache oder den Sprechern gelten. Ich habe deshalb auch gezielt nach stereotypen Vorstellungen vom Sprachverhalten eines Deutschen gefragt. Diese Frage hat sich als besonders plausibel, wenn auch in den Antworten als nicht sonderlich erfreulich erwiesen.

Deutsche werden in ihrem Sprachverhalten durchweg als ,laut im Ton und vorlaut im Benehmen“ angesehen (R. Metrich), und das nicht nur in Frankreich. Aus Dänemark heißt es: „Deutsche reden und lachen sehr laut.“ ( $\mathrm{H}$. Bergenholtz) Finnen betrachten ,deutsche Gesprächspartner als sehr/zu dominant ... mit der Tendenz zum lautstarken Reden“" (so Christopher Schmidt, Turku). Ähnliche Meinungen vermitteln kundige Kollegen aus Italien, der Türkei, Polen, Ungarn, Litauen, Norwegen, Schweden und Spanien. Nur einige Germanistikstudenten in Barcelona meinen, Deutsche redeten eher leise (M. Siguan). Das laute Sprachverhalten wird in verschiedenen Antworten weiter charakterisiert als „grob“, ,überheblich“, ,herrisch“, ,aggressiv“, ,,autoritär“, ,,besserwisserisch“. Hieraus ergibt sich ein Wortfeld unsympathischer Adjektive, das kaum Unterscheidungen nach Ländern und Sprachen zulässt. Einzelne Kolleginnen und Kollegen spezifizieren näher. Vural Ülkü etwa nennt das Sprachverhalten deutscher Beamter in den Behörden, an der

keit, Ordnung, Disziplin, Arbeit, Sachverhalt, amtlich, Behörde, müssen, Einführung (Vural Ülkü); aus Norwegen: energisch, gründlich (Erling Vaagland).

In den Bänden der Comic-Reihe BUR Sturmtruppen (Bonvi), Biblioteca Universale Rizzoli, Milano $1992 \mathrm{ff}$. 
Grenze und in diplomatischen Vertretungen „,sehr grob“, „, beleidigend“ und „erniedrigend“. Spanische Kolleginnen berichten von Eindrücken, die Deutschen seien „laut, sie schreien oft, weil sie sich oft ärgern“ (L. Schilling), und ,[Sie reden so,] dass man, wenn man sie nicht versteht, meinen könnte, sie seien böse." (M. Siguan) Mehrere finnische Kollegen schreiben, auffällig sei für Finnen, dass Deutsche ihre Gesprächspartner oft unterbrechen. Interessant ist, dass Angelika Linke (Zürich) das Gleiche selbst aus der deutschsprachigen Schweiz berichtet. Sie meint, dass dieses Stereotyp sich besonders auf Norddeutsche beziehe, die wegen ihrer ,zackigen“ Aussprache des schweizerdeutschen „Grüetzi“ auch „Grüzzenis“ genannt werden. Ähnliche Einstellungen zum Sprachverhalten von Deutschen gibt es auch in Österreich. Das Adjektiv „dominant“ erscheint in mehreren Antworten, die Frau Kollegin Betten aus Salzburg übermittelt hat.

Weniger negativ ist die aus Ungarn erwähnte Ansicht, Deutsche seien ,allzu präzise, übermäßig genau und meistens korrekt" (Peter Bassola, Budapest). Studenten von Ewa Drewsnowska-Vargáné meinen u. a., ein Deutscher gebe „oft kurze Antworten“, bleibe „beim Thema konsequent.“ Deutsche seien im Umgang ,direkter als z. B. Finnen“(Anniki Koskensalo, Turku), sie seien ,genau und gründlich“ (so aus Norwegen Erling Vaagland, Trondheim). Positiv eingestellt sind auch einige litauische Studenten, die u. a. meinen, ,ein Deutscher denkt nach, dann sagt er treffend und kurz, spricht und argumentiert logisch ..." (Ernesta Raciene). Außer dem laut oder grob sprechenden Deutschen gibt es offensichtlich auch das Stereotyp vom rational bis pedantisch-korrekt argumentierenden Deutschen. Nur vereinzelt werden Deutsche auch für höflich gehalten. Neben viel Kritischem zum Sprachverhalten von Deutschen meint V. Ülkü, von Türken werde andererseits ,die im täglichen Umgang vielfache Verwendung von Begrüßungsformeln Guten Morgen/Guten Tag ... und Dankesworten Danke schön .../Bitte sehr ... als typisch deutsch und nachahmenswert" angesehen.

\section{Erste Folgerungen}

Die zum Teil recht umfangreichen Antwortbriefe und -mails konnte ich nicht ausschöpfen. ${ }^{8}$ Sie können aber sicherlich zu eingehenderen Untersuchungen anregen. Vielleicht werde ich, werden andere Mitarbeiter des IDS bei anderer Gelegenheit noch einmal nachfragen. Das Bild, das sich aus der zusammenfassenden Auswahl ergibt, ist noch ziemlich grobkörnig und in den hervorstechenden Zügen eher unerfreulich. Die negativen Eigenschaften, die der deutschen Sprache und ihren Sprechern zugeschrieben werden, überwiegen. Genau genommen ist freilich die pauschale Zusammenfassung nicht ganz realistisch; denn das dabei entstehende Gesamtbild bietet sich nur aus

\footnotetext{
${ }^{8}$ Die Briefe und E-mails sind im IDS, Mannheim archiviert.
} 
der Innensicht der Außenansichten. Der externe Betrachter fasst ja nicht in dieser Weise zusammen. Der finnische Schüler etwa, der überlegt, ob er Deutsch lernen soll, kennt nicht die Einstellung seiner spanischen oder italienischen Altersgenossen zum Deutschen. Und die junge Französin, für die deutsche Äußerungen hart klingen, weiß nichts von der phonischen Wahrnehmung, die junge Polen oder Türken vom Deutschen haben. Es ist deshalb nötig, den Gesamtbefund und seine Teile noch genauer anzuschauen. Das geschieht in mehreren der anderen Beiträge, in denen jeweils einzelsprachliche Einstellungen zum Deutschen behandelt werden. Einige Meinungen und Bewertungen sind freilich über viele Länder verteilt, u. a. dass Deutsch schwierig ist, die Wortstellung kompliziert, die Wörter lang, die Aussprache hart und dass viele Deutsche bemerkenswert laut sprechen. Den formalen Qualitäten lässt sich durch genauere kontrastive und typologische Untersuchungen nachgehen, welche einen Teil der intuitiven Urteile womöglich auf strukturelle Eigenschaften des Deutschen im Verhältnis zu anderen Sprachen zurückführen. ${ }^{9}$ Was kann man sonst noch tun?

Die deutsche Sprache selbst können wir kaum ändern. Ändern lassen sich aber Meinungen, die auf mangelnder Kenntnis der deutschen Sprache und ihrer Sprecher beruhen. Darauf weisen auch mehrere meiner Korrespondenten hin. Naheliegende Folgerungen aus der meist unerfreulichen ästhetischen Außenbewertung des Deutschen und den oft negativen emotionalen Einstellungen zu dieser Sprache und ihren Sprechern sind:

(1) Die von deutscher Seite geförderte Sprachvermittlung durch GoetheInstitute und DAAD sollte verstärkt, statt immer weiter eingeschränkt werden.

(2) Mehr ausländischen Studierenden und Schülern sollte durch Austausch und Stipendien der Kontakt zu Deutschen ermöglicht werden, damit sie durch unmittelbare Erfahrungen auch einige ihrer sprachbezogenen Vorurteile korrigieren können.

(3) Deutsch sollte in Studium und Unterricht nicht vorwiegend anhand von Alltagsdialogen, Fachtexten und kritischen landeskundlichen Texten präsentiert werden, sondern auch in eingängiger Literatur, darunter vielleicht sogar Lyrik.

Leider lässt sich das kommunikative Verhalten mancher deutscher Geschäftsleute und vieler deutscher Touristen an den Badestränden der Mittelmeerländer und auch anderswo nicht durch staatliche Vorschriften oder gar durch linguistische Forschungen beeinflussen. Die sprachlichen Umgangsformen von Mitarbeitern deutscher Behörden sind jedoch beeinflussbar; auch Beamte haben sich in dieser Hinsicht als lernfähig erwiesen.

\footnotetext{
${ }^{9}$ Siehe hierzu die Beiträge von Werner Abraham und Gisela Zifonun in diesem Band.
} 
Mit Folgerungen wird sich noch die abschließende Podiumsdiskussion (in diesem Band S. $292 \mathrm{ff}$.) zu befassen haben, die dann auch die genaueren Beschreibungen und Analysen der folgenden Vorträge berücksichtigen kann. Als untersuchenswert für die weitere Forschung erscheint mir neben den objektivierbaren linguistischen und bildungspolitischen Gegebenheiten die Frage, inwieweit einige von den der deutschen Sprache und den Deutschen als Sprechern zugeschrieben Eigenschaften auf traditionelle Vorurteile zurückgehen, auf Stereotype, die in der Literatur und in mündlich tradierten Anekdoten zum Teil schon seit Jahrhunderten weitergereicht werden.

Zu berücksichtigen ist hier nicht nur Mark Twain, bei dem es im Übrigen neben dem erwähnten Standardzitat auch für die Grammatik des Deutschen einiges zu holen gibt. Ich denke etwa an die ,denglische" Rede, die er am 4. Juli 1878 vor einem anglo-amerikanischen Studentenclub in Heidelberg gehalten hat und in der er u. a. sagt:

\begin{abstract}
„Wenn aber man kann nicht meinem Rede verstehen, so werde ich ihm später dasselbe übersetz, wenn er solche Dienst verlangen wollen haben werden sollen sein hätte. (I don't know what wollen haben werden sollen sein hätte means, but I notice they always put it at the end of a German sentence - merely for general literary gorgeousness, I suppose.)“10
\end{abstract}

Grammatisch korrekt ist diese Verbsequenz nicht, sie karikiert aber eindrucksvoll die Häufung infiniter Verbformen am Ende mancher deutscher Sätze.

Dass Deutsch im Vergleich zu anderen europäischen Sprachen hart und grob klingt, ist ein Stereotyp, das über die Jahrhunderte in Anekdoten tradiert wird. $\mathrm{Zu}$ den bekannteren gehört die, wonach der polyglotte Kaiser Karl V. deutsch nur sprach, ,wenn er mit jemandem in drohendem oder ziemlich barschen Ton zu reden habe ... weil die Sprache der Deutschen ganz und gar drohend und heftig ist." "Für feinere und höhere Zwecke soll er Spanisch, Italienisch oder Französisch verwendet haben. In Varianten dieser Anekdote, die Harald Weinrich in einer lesenswerten Studie zusammengestellt und erläutert hat, spricht Karl V. deutsch vor allem mit seinen Soldaten und schließlich nur noch mit seinem Pferd. Das Stereotyp vom militärisch harten Klang des Deutschen ist also nicht erst in den beiden Weltkriegen entstanden. Ergänzen möchte ich eine andere alte Anekdote, auf die ich ebenfalls durch Weinrich aufmerksam geworden bin.

${ }^{10}$ A fourth of July oration in the German Tongue, delivered at a banquet of the AngloAmerican Club of Students by the Author of this book. In: A Tramp Abroad, Appendix D. The awful German language. 1880.

"Girolamo Fabrizi d'Acquapendente: De locutione et eius instrumentis. Padua 1601. S. 64; zit. nach Weinrich, Harald: Sprachanekdoten um Karl V. In: Weinrich, Harald: Wege der Sprachkultur. Stuttgart 1985. S. 181-194, S. 182. 
Als es am Hof des polnischen Königs Sigismund um die Sprache Gottes ging, soll ein Spanier verkündet haben, Gott habe auch schon deutsch gesprochen, aber nur, um Adam und Eva mit polternder Stimme aus dem Paradies zu treiben. ${ }^{12}$

Es wäre sicherlich lohnend, weiteren Stereotypen über das Deutsche in anderssprachigen Literaturen nachzugehen. Dass einige Klischees bis in die modernen Comics reichen, wissen wir spätestens seit dem Heft „Astérix chez les Goths"/,Asterix und die Goten“. ${ }^{13}$ Ein lohnendes Feld für linguistisch interessierte Literaturwissenschaftler. Zum Deutschen gibt es glücklicherweise nicht nur negative Vorurteile, von seiner weiterhin attestierten besonderen Eignung für die Philosophie ganz abgesehen. ${ }^{14}$ Eine der schönsten Ansichten von deutscher Sprache findet sich in einem Gedicht des argentinischen Schriftstellers Jorge Luis Borges, das den Hispanophonen unter Ihnen sicherlich bekannt ist. Es heißt „Al idioma alemán“15 und ist zuerst 1972 erschienen. Ich möchte daraus schon wegen meines schlechten Spanisch in deutscher Übersetzung zitieren. Borges spricht darin von der dulce lengua de Alemánia und preist sie als Sprache von Hölderlin, Angelus Silesius, Heine, Goethe und Keller. Wo sonst wird Deutsch als ,süße Sprache' empfunden? Die letzten Zeilen lauten in deutscher Annäherung: ${ }^{16}$

Du, Sprache Deutschlands, bist dein

Hauptwerk: die verschlungene Liebe

der zusammengesetzten Diathesen, die offenen Vokale,

die Laute, die den gelehrten griechischen Hexameter erlauben,

und dein Rauschen von Wäldern und Nächten.

Ich hatte dich einmal. Heute, an der Grenze

zu den müden Jahren, schaue ich dich

weit entfernt wie Algebra und den Mond.

(... lejana como el álgebra y la luna.)

12 Weinrich, a.a. O., S. 183.

${ }^{13}$ Rene Goschinny/Albert Uderzo: Astérix chez les Goths. Paris 1963 ff., deutsche Übersetzung „Asterix und die Goten“. Stuttgart $1970 \mathrm{ff}$.

${ }^{14}$ Deutsch als Philolosophensprache kommt sogar noch in einem modernen brasilianischen Chanson vor: „Filosofar só é possivel em alemáo. “ (Caetano Veloso).

15 Jorge Luis Borges: Al idioma alemán. (El oro de los tigres. 1972) In: Obras poeticas. Buones Aires 1977, S. 393.

${ }_{16}$ Im spanischen Original:

Tú, lengua de Alemánia, eres tu obra capital: el amor entrelazado de las voces compuestas, las vocales abiertas, los sonidos que permiten el estudioso hexámetro del griego y tu rumor de selvas y de noches. Te tuve alguna vez. Hoy, en la linde de los anos cansados, te diviso lejana como el álgebra y la luna. 
Deutsch zu sehen wie „Algebra und den Mond“ ist nicht leicht nachzuempfinden. Aber zusammen mit dem Lob der offenen Vokale und des zusammensetzten Verbalgenus wird daraus eine seltsame, seltene Huldigung der deutschen Sprache. Ob Deutsch süß klingt, hängt für mich vom Sprecher, der Sprecherin und dem jeweils Gesagten ab. Wenn Deutsch von außen her, aus Wahrnehmungsperspektive anderer Sprachen hin und wieder auch als „süß“ oder wie das „Rauschen von Wäldern und Nächten“ empfunden wird, tröstet das mich und vielleicht auch andere hiesige Germanisten über die unserer Sprache und den Deutschen ansonsten stereotyp unterstellte Härte und Grobheit hinweg. 